\title{
Percepción de las relaciones médico-paciente, por parte de los usuarios externos de un departamento de medicina*
}

\author{
Perception of doctor-patient relationship by external users of a medicine department
}

\author{
Claudia Ramos-Rodríguez ${ }^{1}$ \\ ${ }^{1}$ Médico-Cirujana, Facultad de Medicina de San Fernando. \\ * Trabajo de Tesis para optar el título de Médico-Cirujano, Facultad de Medicina - Universidad Nacional Mayor de San Marcos. Lima, Perú.
}

\begin{abstract}
Resumen
Introducción: La relación médico paciente, la mayor potencia recuperadora de la salud, está hoy amenazada por los cambios que afectan la práctica médica. Objetivos: Determinar la percepción, por parte de los usuarios de consulta externa, de la calidad de su relación médico-paciente y su asociación con las características del médico y el paciente. Diseño: Estudio observacional, tipo encuesta. Lugar: Departamento de Medicina, Hospital Nacional Daniel Alcides Carrión, Callao, Perú, hospital docente. Participantes: Pacientes de consulta externa de medicina. Intervenciones: Un cuestionario semiabierto fue aplicado a los pacientes seleccionados por muestreo sistemático, al salir del consultorio. Principales medidas de resultados: Calidad de la relación médico-paciente. Resultados: De 168 pacientes, 39,9\% ya había acudido a otro lugar por su problema de salud, $51,2 \%$ fue atendido por primera vez por determinado médico, 92,3\% tuvo una adecuada relación médico-paciente y $89,3 \%$ afirmó estar muy satisfecho. Se observó que mayor es el grado de satisfacción de la consulta a mejor percepción de la calidad de relación médico-paciente ( $p$ $<0,001,0 R 61,2)$; ambas se asociaron a la intención de recomendación del médico (OR 298 y OR 48, respectivamente). Conclusiones: Se encontró una adecuada relación médico-paciente en $92,3 \%$ de los pacientes encuestados, y $89,3 \%$ afirmó estar muy satisfecho con la atención médica.

Palabras clave: Médicos; pacientes; atención médica.
\end{abstract}

\begin{abstract}
Introduction: Physician-patient relationship is the most important power for health recovery. Nevertheless it appears threatened by the many changes affecting today's medical practice. Objectives: To determine patient's perception on the quality of the doctor-patient relationship and its association with physician's and patient's characteristics. Design: Observational study, inquiry type. Setting: Department of Medicine, Hospital Nacional Daniel Alcides Carrion, Callao, Peru, a teaching hospital. Participants: Medicine outpatient patients. Interventions: A semi-opened questionnaire with 21 questions was applied to systematically selected patients when leaving the doctors' office. Main outcome measures: Quality of doctor-patient relationship. Results: One hundred and sixty-eight patients were inquired, 39,9\% had been previously attended in other place for the same health problem, $51,2 \%$ were attended for the first time. An adequate doctor-patient relationship was found in $92,3 \%$ and $89,3 \%$ were very satisfied. The quality of doctorpatient relationship perceived was strongly associated to medical attention satisfaction ( $p<0,001,0 R 61,2)$. Both were associated with recommending the doctor (OR 298 and OR 48 respectively). Conclusions: We found an adequate doctor-patient relationship in $92,3 \%$, and $89,3 \%$ said they were very satisfied with the medical attention.

Key words: Physicians; patients; medical care.
\end{abstract}

\section{INTRODUCCIÓN}

En el acto médico, como en todo contacto humano, se crea una relación interpersonal muy importante ${ }^{(1,2)}$, en lo que se denomina relación médico-paciente. Sus efectos habrán de evidenciarse en ambos participantes. Para el paciente, las consecuencias inmediatas de una buena consulta médica serán el consentimiento del plan terapéutico propuesto, la satisfacción con la atención recibida, la recolección mnésica de lo que se dijo y algún cambio en el grado inicial de preocupación que lo llevó al consultorio. Los efectos intermedios serán la adherencia al tratamiento propuesto $y$, a largo plazo, el cambio en su salud y en su 'entendimiento' (3). Para el médico, la consecuencia inmediata será una experiencia placentera o no, que influirá en el estilo de sus posteriores consultas; el efecto intermedio será el retorno del paciente a una segunda cita (continuidad del manejo); y, a largo plazo, la satisfacción con su trabajo (3), lo cual les hace médicos más efectivos (4-6). Además de disminuirles los litigios por malapraxis ${ }^{(7)}$.

La percepción de desinterés del médico, de su prisa o falta de calor humano, generalmente contribuyen a que el paciente no aporte los datos que se le piden o los dé con similar espíritu de salir del paso ${ }^{(1)}$. Como consecuencia de esto, se elaborará una historia clínica incompleta, que llevará al planteamiento de un diagnóstico diferencial inadecuado. Por esto, el diagnóstico probablemente será erróneo y el plan terapéutico inadecuado. Finalmente, los resultados médicos no serán óptimos. Esto iniciará un círculo vicioso, porque el paciente perderá aún más la confianza en el médico y tal vez, también, en su gremio.

Pero, ¿cómo se logra establecer o mantener la confianza? Muchas recomendaciones son dadas. Para comenzar, es fundamental que el profesional se presente con una vestimenta apropiada e higiene cuidadosa; igual de pulcro y privado debe ser el lugar donde se realice toda atención ${ }^{(8,9)}$. Debe saludar a su paciente por su nombre, ya que el primer acto del tratamiento es extender la mano al paciente ${ }^{(2)}$. Debe dar a conocer su nombre y cargo ${ }^{(10)}$. Es recomendable que el médico se siente frente al enfermo, le mire a la cara, preferentemente a los ojos ${ }^{(11)}$. El vocabulario con el que se dirige a su paciente y a los demás también debe ser depurado ${ }^{(12)}$.

Si bien lo anterior es importante, talvez lo sean aún más sus actitudes. Así, el médico debe desarrollar su capacidad de empatía ${ }^{(13-15)}$, la cual también gratifica al médico y le hace mejorar como profesional y como persona ${ }^{(16)}$. Debe poseer y expresar humanidad ${ }^{(17,18)}$. Debe dar siempre la sensación de seguridad ${ }^{(19)}$ y lograr la confianza del paciente en su persona y en 
su profesionalismo ${ }^{(8)}$. Calmar la ansiedad con la que viene ${ }^{(20)}$. Es indispensable la disponibilidad a escuchar ${ }^{(19,21)}$, dejar que el enfermo exponga libremente sus molestias (19), asegurando la falta de interrupciones. La participación proporcional de ambas partes es también necesaria ${ }^{(22)}$. Durante la consulta, se debe intercambiar los puntos de vista acerca de la enfermedad del paciente y no dejar discrepancias sin resolver ${ }^{(4,19)}$. Siendo el paciente partícipe de las decisiones terapéuticas ${ }^{(7,10,23)}$. Además, el médico debe ser veraz ${ }^{(10)}$, educador y alentador ${ }^{(24)}$.

Sin embargo, en medicina, se han producido cambios externos al profesional, en el tiempo que nos toca vivir, alterando la relación médico-paciente ${ }^{(25)}$. Aquella está sufriendo impersonalización, expresándose en la sustitución de los términos médico y paciente por los de proveedor y cliente $(23,26,27)$. No al mismo ritmo, se han dado cambios de las expectativas de los pacientes, sobre lo que tienen que recibir de 'sus' médicos ${ }^{(27)}$. La casi extinción del 'médico tratante' no hace fácil la continuidad del manejo, tan valorada. Además, los profesionales médicos han perdido 'estatus', pudiendo haber influido en esto los litigios por malapraxis y la paga inadecuada. Y como si esto fuera poco, el tiempo de la consulta y la visita médica son cada vez más cortas, en buena parte por la productividad exigida hoy. Por otra parte, los pacientes adquirieron 'empoderamiento' acerca de su salud, tienen acceso a información médica en internet y otros medios de comunicación masiva.

$\mathrm{Al}$ mismo tiempo en que se produce la situación descrita en el párrafo anterior, la educación médica en las aulas aún reproduce una imagen omnipotente y autosuficiente del médico, que nada tiene que ver con la realidad ${ }^{(20,28)}$.

El objetivo del presente estudio fue determinar la percepción, por parte de los usuarios externos del Hospital Nacional Daniel Alcides Carrión (HNDAC), de la calidad de sus relaciones médico-paciente (RMP) y su asociación con las características del médico y el paciente.

\section{MÉTODOS}

Se realizó un estudio de tipo observacional. El tipo de muestreo utilizado en este trabajo fue muestreo no probabilístico incidental. Se calculó una muestra de 187 pacientes (IC $=95 \%, 6 \%$ de error absoluto), que acudieron a los consultorios externos de los 11 servicios del Departamento de Medicina del Hospital Nacional Daniel Alcides Carrión, sede docente de la UNMSM, un día elegido al azar, en el año 2006. Los pacientes elegidos, eran encuestados al salir del consultorio, si no cumplían con los criterios de exclusión y si previamente habían aceptado verbalmente (en la sala de espera), luego de leer el consentimiento informado de la ficha informativa del estudio.

Se usó un cuestionario estructurado semiabierto, llamado PREMEPA (percepción de la relación médico-paciente), diseñado para el estudio, cuyo bloque 1 fue validado por juicio de expertos ( 5 especialistas en el área), y evaluado en confiabilidad (consistencia interna) por la fórmula de KüclerRicharson ( $K=0,833$, índice de fiabilidad de 91,3\%), después de dos aplicaciones piloto, con un total de 70 usuarios de consultorios externos del Departamento de Cirugía y Ginecoobstetricia del mismo Hospital Daniel Alcides Carrión.

El Bloque I estuvo conformado de 14 preguntas: La primera era una pregunta abierta que tenía como objetivo averiguar si consultó ayuda previa antes por el mismo problema, que ahora lo trae al consultorio. Las preguntas de la 2 a la 9 eran de opción múltiple (4 opciones) y se evaluó por medio de una escala de Likert modificada (valores del 0 al 3) las actitudes y características del profesional percibidas en la consulta, en cuanto a respeto y cortesía, disponibilidad de escuchar, comprensión, sensibilidad humana, así como el grado de confianza en su capacidad profesional y la participación del paciente en la consulta. Los valores obtenidos en estas preguntas y las preguntas dicotómicas 10 a la 13 (valores 0 y 3 ) serían luego convertidas a una variable cualitativa llamada 'calidad de relación médico-paciente', mediante sumatoria de sus puntajes, ubicando la relación médico-paciente evaluada dentro de una de tres categorías. Se definió una relación médico-paciente adecuada como aquella que lograba un puntaje mayor o igual a 15; relación médico-paciente inadecuada, aquella con puntaje menor o igual a 8 ; $y$, relación médico-paciente medianamente adecuada, las de puntaje intermedio.

La pregunta 14, acerca de satisfacción del paciente con la atención médica, fue evaluada mediante la escala vigesimal. Este valor también fue transformado a grados de satisfacción, donde muy satisfecho era un puntaje mayor de 15 , poco satisfecho uno menor de 10 y medianamente satisfecho, el puntaje intermedio.
El Bloque II fue titulado 'Datos generales del médico' e incluía sexo, grupo etáreo, número de consultas anteriores con ese médico y elección o no del médico.

El Bloque III fue titulado 'Datos generales del paciente' e incluía sexo, edad, tipo de paciente (nuevo, continuador) y grado de instrucción.

Con los datos obtenidos, se realizó análisis univariado, análisis bivariado (chi-cuadrado o prueba exacta de Fisher y regresión logística simple), utilizando el paquete estadístico SPSS 11,0. Se consideró una diferencia estadísticamente significativa los valores $p<0,05$, para un nivel de significancia de $95 \%$.

\section{RESULTADOS}

Se encuestó un total de 168 pacientes. Las características socio-demográficas de los pacientes son detallados en la Tabla $1 \mathrm{y}$, las de los médicos, en la Tabla 2.

Tabla 1. Características generales de los pacientes.

\begin{tabular}{llrl}
\hline \multicolumn{1}{c}{ Variable } & & $\mathrm{N}^{\circ}$ de pacientes \\
\hline Sexo & Masculino & 65 & $(38,7 \%)$ \\
& Femenino & 103 & $(61,3 \%)$ \\
Edad & Adulto joven & 78 & $(46,4 \%)$ \\
& Mediana edad & 50 & $(29,8 \%)$ \\
& Adulto mayor & 40 & $(23 \%)$ \\
Tipo de paciente & Nuevo & 80 & $(47,6 \%)$ \\
& Continuador & 88 & $(52,4 \%)$ \\
Grado de Instrucción & Analfabeto & $10,6 \%)$ \\
& Primaria incompleta & 21 & $(12,5 \%)$ \\
& Primaria completa & 15 & $(8,9 \%)$ \\
& Secundaria incompleta & 25 & $(14,9 \%)$ \\
& Secundaria completa & 49 & $(29,2 \%)$ \\
& Superior incompleta & 16 & $(9,5 \%)$ \\
& Superior completa & 41 & $(24,4 \%)$ \\
\hline
\end{tabular}

El 52,4\% de los pacientes ya había acudido a algún servicio del hospital, 47,6\% acudía a los consultorios externos del Departamento de Medicina por primera vez, para el problema de salud que les hizo ir ese día a consultorio; $74,4 \%$ afirmó no haber elegido al médico que le atendió ese día en la consulta.

Tabla 2. Características generales de los médicos.

\begin{tabular}{llrl}
\hline \multicolumn{1}{c}{ Variable } & & $\mathrm{N}^{\circ}$ de médicos \\
\hline Sexo & Masculino & 129 & $(76,8 \%)$ \\
& Femenino & 39 & $(23,2 \%)$ \\
Edad & Adulto joven & 64 & $(38,1 \%)$ \\
& Mediana edad & 79 & $(47 \%)$ \\
& Adulto mayor & 25 & $(14,9 \%)$ \\
Elegidos por los pacientes & No & 125 & $(74,4 \%)$ \\
& $\mathrm{Si}$ & 43 & $(25,6 \%)$ \\
$\mathrm{N}^{\circ}$ de consultas anteriores & 0 consultas & 86 & $(51,2 \%)$ \\
& 1 a 3 consultas & 39 & $(23,2 \%)$ \\
& más de 4 & 43 & $(25,6 \%)$ \\
\hline
\end{tabular}


En la Tabla 3 se aprecia las características que nos proporcionaron los puntajes para calificar las relaciones médico-paciente percibidas. Como se puede apreciar, el puntaje promedio obtenido en cada ítem estaba entre 2,65 y 2,88, en la escala del 0 al 3 .

En el rubro participación del paciente, debemos mencionar que $2,4 \%$ y $1,2 \%$ de los pacientes refirieron, respectivamente, que entendieron nada o muy poco de la explicación del diagnóstico; 2,4\% y 6,5\% refirieron que entendieron nada o muy poco la explicación de su tratamiento, respectivamente; $8,9 \%$ no estaba conforme con el tratamiento, pero $73,3 \%$ no pidió otras opciones.

Se halló una relación médico-paciente adecuada en $92,3 \%$ de las relaciones médico-paciente evaluadas, $6 \%$ tuvo una relación médico-paciente medianamente adecuada y $1,8 \%$ una relación médico-paciente inadecuada. El promedio del puntaje de relación médico-paciente fue 19 , en el presente estudio. De la misma forma, se encontró poca satisfacción en $6 \%$, mediana satisfacción en 4,8\% y mucha satisfacción en $89,3 \%$. La media del puntaje dado fue 17. El 89,3\% afirmó que recomendarían al médico que los atendió en esa oportunidad, con sus familiares o amigos.

Se observó que mayor es el grado de satisfacción de la consulta a mejor percepción de la calidad de relación médico-paciente; de la misma forma, que la calidad de relación médico-paciente hallada y el grado de satisfacción de la consulta médica se asociaron a la intención de recomendación del médico (Tablas 4 y 5 ).

Tabla 4. Asociación entre percepción de la calidad de la relación médico-paciente y las variables del estudio.

\begin{tabular}{lr}
\hline \multicolumn{1}{c}{ Variable } & Valor $p$ \\
\hline Grado de instrucción del paciente & 0,25 \\
Grupo etáreo del paciente & 0,02 \\
Grupo etáreo del médico & 0,4 \\
Concordancia de grupo etáreo del médico y paciente & 0,59 \\
Género del médico & 0,15 \\
Concordancia de género del médico y paciente & 0,15 \\
Continuidad del manejo médico & 0,30 \\
Elección del médico antes de la consulta & 0,02 \\
Intención de recomendación del médico & $<0,001$ \\
Satisfacción de la consulta & $<0,001$
\end{tabular}

Tabla 5. Regresión logística entre las variables del estudio.

\begin{tabular}{lrc}
\hline \multicolumn{1}{c}{ Variable } & OR & IC (95\%) \\
\hline Calidad RMP vs. satisfacción en la consulta & 61,2 & $14,0-267,3$ \\
Calidad RMP vs. intención de recomendación & 298 & $33-2681$ \\
Calidad RMP vs. grupo etáreo & 4,2 & $1,1-16,1$ \\
Calidad RMP vs. elección del médico & 1,3 & $1,2-1,5$ \\
Satisfacción vs. intención de recomendación & 48 & $13,4-171,8$ \\
\hline
\end{tabular}

RMP: relación médico-paciente.

Tabla 3. Actitudes de médicos y características de la relación médico-paciente.

\begin{tabular}{lcccrcr}
\hline & \multicolumn{5}{c}{$\mathrm{N}^{\circ}(\%)$ de pacientes } & Puntaje promedio \\
\cline { 2 - 5 } & No $(0)$ & Muy poco (1) & Medianamente $(2)$ & Totalmente (3) & \\
\hline Respeto y cortesía & $2(1,1)$ & $7(4,2)$ & $13(7,7)$ & $146(86,9)$ & 2,8 \\
Disponibilidad a escuchar & $0(0,0)$ & $6(3,6)$ & $9(5,4)$ & $153(91,1)$ & 2,88 \\
Comprensión & $0(0,0)$ & $9(5,4)$ & $20(11,9)$ & $139(82,7)$ & 2,77 \\
Sensibilidad & $1(0,6)$ & $3(1,8)$ & $36(21,4)$ & $128(76,2)$ & 2,73 \\
Confianza & $4(2,4)$ & $2(1,2)$ & $42(25,0)$ & $120(71,4)$ & 2,65 \\
Participación & $5(3,0)$ & $6(3,6)$ & $17(10,2)$ & $140(84,0)$ & 2,74 \\
\hline
\end{tabular}

El haber elegido o no al médico no se asoció a la intención de recomendar al médico a amigos o familiares $(p=0,110)$, pero la frecuencia de haber elegido al médico y desear recomendarlo fue un poco mayor $(95,4 \%)$ que cuando no fue elegido el médico por el paciente $(87,2 \%)$. Haber elegido o no al médico también se asoció significativamente a la calidad percibida de la relación médico-paciente $(p=0,02)$.

\section{DISCUSIÓN}

Se ha hallado datos muy alentadores en el presente estudio sobre la tan importante relación médico-paciente. Si bien esta relación, tal como fue definida, aquí no fue evaluada en otras investigaciones, las características empleadas sí lo fueron.

Fernández, en el hospital militar geriátrico de Lima, en el 2003, encontró que 74\% de pacientes describieron a su médico como amable, un porcentaje menor a lo encontrado en nuestro estudio, en que $86,9 \%$ contestó que el médico fue muy respetuoso y cortés ${ }^{(29)}$.

En la tesis de Mulanovich, realizada en 46 pacientes internados en los hospitales limeños, el promedio del puntaje dado a la disponibilidad de escuchar por parte de su médico fue 4,03, en una escala del 1 al 5 . En empatía del médico, el puntaje fue 4,63 , trato como persona 5,0 y confianza en el médico 4,47, en promedio ${ }^{(11)}$; mientras, en nuestro estudio obtuvimos valores de $2,88,2,77$ y 2,65 , sobre 3 , respectivamente.

Hay que destacar que algunas de estas características fueron alteradas por diversos factores del médico y del paciente. Epstein, en su investigación, donde se grabó 100 videos de consultas médicas de internistas en EE UU, vio que la respuesta empática de médicos mujeres fue significativamente más frecuente que entre los galenos varones ${ }^{(30)}$.

Miettola, en su estudio realizado en Finlandia, anotó que los estudiantes de medicina del primer año, en su segundo día de clases, también percibieron que los médicos usaban diferentes formas de comunicación con los pacientes, dependiendo del paciente, añadiendo que si bien la comunicación con pacientes ordinarios era empática, se tornaba más distante en casos especiales y críticos, como con los niños, retardados mentales, ancianos, pacientes socialmente marginados y con pacientes en la sala de emergencia ${ }^{(31)}$.

Acerca de la confianza en el médico, una de las características principales de una adecuada relación médico-paciente, fue declarada como plena por $71,4 \%$ de los entrevistados, cifra muy cercana a la encontrada por Andía en un estudio en Cusco, Perú, en un hospital del seguro social, año 2002 , donde $62,2 \%$ de los 91 usuarios encuestados respondió tener plena confianza y seguridad en el médico ${ }^{(32)}$. Keating, en un cuestionario telefónico de 8 ítems aplicado a 424 pacientes, encontró que $79 \%$ afirmaron sentir plena confianza en el médico que los atendió y los factores asociados a esta respuesta fueron la participación del paciente en la toma de decisiones, duración de la consulta -tal como la deseaba el paciente-, entre otros ${ }^{(33)}$.

En un estudio nacional de Shenolikar, en Gran Bretaña, en 2004, se encontró que el sentimiento de vulnerabilidad del paciente influye grandemente en el grado de confianza a lograrse dentro de la relación médico-paciente ${ }^{(34)}$. De forma similar, Kroenke, en un cuestionario telefónico realizado en 1117 pacientes, encontró una relación significativa entre la confianza en el médico y ser evaluado por enfermedades serias ${ }^{(35)}$.

La participación también fue mencionada en el estudio de Fernández, en donde se señala que los médicos les explicaron su diagnóstico y tratamiento a $85 \%$ y $88 \%$ de los pacientes entrevistados, respectivamente ${ }^{(29)}$. En el estudio de Andía, $67,1 \%$ de los pacientes dijeron estar muy satisfechos de manera general y satisfechos con la explicación del médico acerca de su enfermedad (32). En una encuesta en 370 usuarios externos de un hospital de Iquitos, los pacientes opinaron que en $85 \%$ de las veces el médico 
les hizo entender su problema de salud y que en $72 \%$, les explicó su tratamiento y cuidados a seguir ${ }^{(36)}$. Refiriéndose al mismo tema, Mulanovich concluye que existe menos satisfacción con la información recibida sobre la enfermedad, que con la conducta del médico o con el aspecto afectivo de la relación médico-paciente ${ }^{(11)}$.

Cooper encontró que las mujeres médicos dan consultas con mayor participación del paciente que los profesionales varones (37). En nuestro estudio, el género del médico no se asoció a la calidad percibida de relación médico paciente; sin embargo, hubo una tendencia en el mismo sentido. Otros factores señalados en la participación fueron el estado emocional y grado de educación, según Back ${ }^{(38)}$. Cooper afirmó que los pacientes con etnicidad concordante a la de sus médicos participan más en la consulta y que los pacientes afro-americanos tienen, significativamente, menos participación en la entrevista que los blancos (37). En nuestro estudio, realizado en una población predominantemente mestiza, estas diferencias no fueron evaluadas.

No hubo asociación significativa entre el número de consultas previas y la calidad de relación médico-paciente en el presente estudio, a diferencia de lo que se halló en el estudio de Von Bultzingslowen, en el cual pacientes crónicos respondieron en gran mayoría que preferían un 'doctor personal', conocido por largo tiempo, ya que sentían más comprensión y empatía ${ }^{(39)}$. Miettola también señaló que, cuando los pacientes conocen al médico, parecen ser más abiertos ${ }^{(31)}$.

El promedio del puntaje de satisfacción en esta pesquisa fue 17 sobre 20 puntos. En el estudio de Sulmasy, en el 2002, en 84 pacientes terminales hospitalizados, fue 4,39 (en una escala del $1 \mathrm{al}$ 5) ${ }^{(40)}$. Un poco menor en el Cusco, Andía afirmó que el promedio fue 3,67, en una escala del 1 al 5 (32).

En el estudio del hospital Cesar Garaya García, de Iquitos, se menciona que 65\% de los pacientes que respondieron estuvo satisfecho de su atención, de manera general ${ }^{(36)}$. Tsuchida, en Lima, en el 2003, en las salas de hospitalización halló 82,8\% ${ }^{(41)}$, cifra más cercana al $89 \%$ hallado en el presente estudio.

Según Frostholm, en Dinamarca, en el 2005, los pacientes que consultaron un médico más experimentado, entrenados en el trato con el paciente y de sexo femenino, tenían más probabilidad de estar más satisfechos ${ }^{(42)}$. Vedsted halló, a su vez, en el 2002, que los pacientes con niveles socioeconómicos más altos mostraban índices de satisfacción más bajos ${ }^{(43)}$.

En una encuesta telefónica hecha en 1816 pacientes, se evaluó satisfacción de la atención en general y se halló que era mayor entre mayores de 40 años. Cooper también halló mayores porcentajes de satisfacción entre los pacientes con educación universitaria 37. Lo mismo fue visto en un hospital de Lima, en el 2000, aplicado a 97 personas, donde las calificaciones fueron más bajas entre los usuarios con educación superior ${ }^{(44)}$. En el estudio de Saco, en Cusco, donde se tuvo 360 participantes de primer nivel de atención, no se encontró esta relación ${ }^{(45)}$. En nuestra investigación, la satisfacción se asoció significativamente a la percepción de la calidad de la relación médico-paciente. Es decir que, las características y actitudes que evaluamos en ella realmente son importantes para el paciente y, por lógica, se asociarán a todos aquellos beneficios que la satisfacción de la consulta médica les trae, motivo por el cual deben ser tenidos en cuenta en cada acto médico que realicemos. De igual modo, se asoció la intención de recomendación del médico y la satisfacción, confirmando lo que también se observó en el estudio de Andía ${ }^{(32)}$.

Muchas de las variables que afectan a las características involucradas en la relación médico-paciente no pueden ser modificadas. Por lo que, luego de la exposición de los datos previos, queda comentar los esfuerzos que pueden ser realizados para mejorar esta vital relación. En la universidad de Minessota, EE UU, se instauró el 'programa de mentores', donde los estudiantes del $3^{\text {er }}$ año de medicina pasan su tiempo libre durante 9 meses con un mentor, cuidadosamente seleccionado, que es ejemplo de profesionalismo y les da la oportunidad de reflexionar sobre las experiencias del día en la practica médica compartida con sus pupilos ${ }^{(46)}$. Este programa tiene como objetivo lograr mejorar las habilidades y reforzar valores en los alumnos, para que logren mejor relación médico-paciente en su futura vida profesional, ya que es sabido que las habilidades de comunicación son un aspecto vital que pueden ser desarrolladas en los estudiantes y que, por el contrario, sin ser incentivadas. se podrían perder ${ }^{(47)}$. Si bien es verdad que se puede guiar a los alumnos en el camino a ser mejores profesionales, los valores de los futuros médicos fueron forjados mucho antes de ingresar a la facultad. Más aún, con la gran densidad poblacional médica en el mercado laboral actualmente en el país, también se incrementa el riesgo de profesionales con características personales más vulnerables. Por lo que la selección debe ser adecuada, no solo basada en conocimientos, sino también en las aptitudes de los postulantes a carreras médicas. La constante evaluación del desenvolvimiento de los médicos por medio de las opiniones de los pacientes y el reconocimiento de aquellos más queridos por los pacientes, lo cual los estimulará a ser mejores cada día, son medidas no difíciles de aplicar y que podría mejorar la calidad de atención y la salud de nuestra población.

\section{AGRADECIMIENTOS}

Al Dr. Alberto Perales Cabrera, Director del Instituto de Ética de la Facultad de Medicina de San Fernando y Profesor principal de la UNMSM, asesor principal de la Tesis a la cual hace referencia el presente trabajo.

Al Consejo Superior de Investigaciones de la universidad y la Unidad de Investigación de la Facultad, quienes apoyaron el financiamiento de éste proyecto.

Al Hospital Nacional Daniel Alcides Carrión, por su apoyo constante a la investigación.

\section{REFERENCIAS BIBLIOGRÁFICAS}

1. Seguín C. Introducción a la medicina psicosomática.1 $1^{\text {a }}$ Edición. Lima: Ed Tscheuch; 1947: 140-2.

2. Laín Entralgo P. La Relación Médico-Paciente: Historia y teoria. $1^{a}$ Edición. Madrid: Ed. Castilla; 1964:15-26.

3. Pandleton D, Schofield T, Tate P, Havelook P. The new consultation: developing doctor-patient

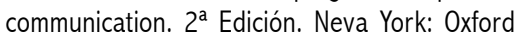
University Press; 2003:38-45.

4. Valdivia R, Méndez S. Factores asociados al síndrome burnout en médicos y enfermeras del hospital nacional sur este de EsSalud del Cusco. Situa. 2003;12(23):11-22.

5. Veerbek J, Boer A, Von der Weide W, Piirainen H, Anema J, Van Amstel R, Hariog F. Patient satisfaction with occupational health physicians, development of a questionnaire. Occup Envioron Med. 2005;62:119-23.

6. Braunwald E, Hauser S, Fauci A, Longo D, Kasper D, Jameson J. Principios de Medicina Interna Harrison 15' Edición. Madrid: Ed Mac Graw Hill; 2002:1-2.

7. Epstein R, Hundert E. Defining and assessing professional competence. JAMA. 2002;257(2):22633.

8. Celeno C. Semiología médica. $3^{\text {a }}$ Edición. México DF: Mac Graw Hill Interamericana; 1999:19-23.

9. Dapper docs: scrubs versus shirt and tie: physicians think patients care about the way they dress. Minn Med. 2007;90(8):10-1. 
10. Kaplan H, Sadock B, Grebb J. Sinopsis de psiquiatría: Ciencias de la conducta y psiquiatría clínica. $7^{a}$ edición. Buenos Aires: Ed Médica Panamericana; 1996:1-15.

11. Mulanovich V. Relación médico-paciente en servicios docentes de hospitalización del servicio de medicina. Tesis de Bachiller para optar por el título de médico-cirujano. Universidad Peruana Cayetano Heredia. Lima, Perú; 1989.

12. Andreassen H, Trondse M, Kummervold P, Gammon D, Hjortdahl P. Patient who use emediated communication with their doctor: New constructions of trust in the patientdoctor relationship. Qualitative Health Research. 2006;16(2):238-48.

13. Larson E, Yao X. Clinical empathy as emotional labor in the patient-physician relationship. JAMA. 2005;239:1100-6.

14. Kraytman M. El diagnóstico a través de la historia clínica. $1^{a}$ Edición. Madrid: Ed IDEPSA; 1995.

15. Myerscough P. Talking with patient. $1^{\text {a }}$ Edición. Nueva York: Oxford University Press; 1989:19-23.

16. Coulehan, J. "Let me see if I have this right..... Words that help build empathy". Ann Intern Med. 2001;135:221-6.

17. Seguin A. Amor y psicoterapia. $1^{\text {a }}$ edición. Buenos Aires: Ed. Paidós; 1963:9-15.

18. Delgado H. El médico, la medicina y el alma. $1^{\text {a }}$ edición. Madrid: Editorial Paz Montalvo; 1952:13-8.

19. Surós A, Surós J. Semiología médica y técnica exploratoria. $8^{a}$ edición. Barcelona: Ed. Masson; 2001:20-4.

20. Tate P. The doctor's comunication handbook. $4^{\mathrm{a}}$ edición. Londres: Medical Press; 2003:24-30.

21. Le Fanu J. Doctor's diary. $1^{\text {a }}$ Edición. Londes: Ed. Robbinson; 1996:15-20.

22. Stewart M, Brown J, Boon H, Galajda J, Meredith L, Sangster M. Evidence on patient-doctor communication. Cancer Prev Control. 1999;3:2530.

23. Santilla, C. Impacto de la reforma en salud en el Perú sobre el acto médico. Pediátrica. 2001;4(2):48-53.

24. Johnsen J, Makerud K. What did the doctor say- what did the patient hear? Operational knowledge in clinical communication. Fam Pract. 1997;14:382-6.

25. Mohammadreza H. Physician empathy: definition, components, and relationship to gender and specialty. Am J Psychiatry. 2002;159:1563-9.
26. Vera-Bejar E. La enseñanza ética en el pre-grado. Boletín Soc Per Med Interna. 2001;14(2):10-4.

27. Potter S, Mc Kinlay J. From a relationship to encounter: an examination of longitudinal and lateral dimensions in the doctor-patient relationship. Social Science and Medicine. 2005;61:465-79.

28.Zárate E. Los derechos de los pacientes y el consentimiento informado en el Perú. SITUA. 2001;23:4-10.

29. Fernández J. Grado de satisfacción del usuario externo en los servicios de consulta externa del Hospital Militar Geriátrico. Tesis para optar por el título de especialista en medicina integral y gestión en salud. Facultad de Medicina, Universidad Nacional Mayor de San Marcos. Lima, Perú; 2003.

30. Epstein R, Bortell F. Pudor, honor, and autoridad: the evolving patient-physician relationship in Spain. Patient Education and Counselling. 2001;45:51-7.

31. Miettola J, Mantyselka P, Vaskilampi T. Doctorpatient interaction in Finnish primary health care as perceived by first year medical students. BMC Medical Education. 2005;5:34-40.

32. Andia C, Pineda A, Sottec V, Santos J, Molina M, Romero Z. Satisfacción del usuario de los servicios de consulta externa del hospital I Espinal. SITUA. 2002;20:18-22.

33. Keating N, Gandhi T, Orav EJ, Bates D, Ayanian J. Patient characteristics and experiences associated with trust in specialist physician. Arch Intern Med. 2004;164:1015-20.

34. Shenolikar E. How patient-physician encounters in critical medical situations affect trust: results of a national survey. BMC Health Services Research. 2004:4:24-30.

35. Kroenke J. Difficult patient encounters in the ambulatory clinic. Arch Intern Med. 1999;159(10):1069-75.

36. Ministerio de Salud del Perú. Encuesta de satisfacción de usuarios del Hospital César Garayar García [Informe técnico]. Lima: MINSA, Unidad de gestión de calidad en salud; 2005.

37. Cooper L, Gallo J, Gonzales J, Vu HT, Powe N, Nelson C, Ford D. Race, gender and partnership in the patient-physician relationship. JAMA. 1999;262:583-9.

38. Back A, Antold R, Baile W, Tulsky J, Fryer-Edwards K. Approaching difficult communication task in oncology. CA Cancer J Clin. 2005;55:164-77.
39. Von Bultzingslowen T. Patients' view on interpersonal continuity in primary care: a sense of security based on four core foundations. Fam Pract. 2005;22(2):254-9.

40. Sulmasy D, Mc llvane J. Patient's ratings of quality and satisfaction with care at the end of life. Ach Intern Med. 2002;162:2098-104.

41. Tsuchida M, Bandres M, Guevara X. Nivel de satisfacción general y análisis de la relación médico-paciente de los médicos en entrenamiento en las salas de hospitalización de medicina interna. Rev Med Hered. 2003;14:175-80.

42. Frostholm L, Fink P, Oernboel E, Christensen KS, Toft T, Olesen F, Weinman J. The uncertain consultation and patient satisfaction: the impact of patients' illness perceptions and randomized controlled trial on the training of physicians' communication skills. Psychosomatic Medicine. 2005;67:897-905.

43. Vedsted P, Mainz J, Lauritzen T, Olesen F. Patient and GP agreement on aspects of general practice care. Fam Pract. 2002;19:339-43.

44. Manzoni GC, Torelli P. The patient-physician relationship in the approach to therapeutic management. Neurol Sci. 2007;2:130-3.

45.Saco S, Farfán R, Andrade V, Martínez C. Diagnóstico basal de la calidad de atención de los establecimientos de salud de la red de Quispicanchi y Acomayo desde la perspectiva del usuario. SITUA. 2001;19:24-35.

46. Bell H. The mentor's image, observing otrher is the best way for students to learn professionalism. Minn Med. 2007;90(8):24-30.

47. Aspergren K, Lonberg P. Which basic communication skills in medicine are learnt spontaneously and which need to be taught and trained? Medical Teacher. 2005;27(6):539-43.

Manuscrito recibido el 7 de marzo de 2008 y aceptado para publicación el 15 de febrero de 2008.
Correspondencia:
Claudia Carolina Ramos-Rodríguez
Facultad de Medicina - UNMSM
Lima 1, Perú
Correo-e: clacrr@hotmail.com 